\title{
Pectoral Vessel Density and Early Ultrastructural Changes in Broiler Chicken Wooden Breast Myopathy
}

Sihvo, H. -K.

2018-05

Sinvo , H -K , Airas , N , Linden , J \& Puolanne , E 2018 , ' Pectoral Vessel Density and Early Ultrastructural Changes in Broiler Chicken Wooden Breast Myopathy ' , Journal of Comparative Pathology, vol. 161 , pp. 1-10 . https://doi.org/10.1016/j.jcpa.2018.04.002

http://hdl.handle.net/10138/327489

https://doi.org/10.1016/j.jcpa.2018.04.002

cc_by_nc_nd

acceptedVersion

Downloaded from Helda, University of Helsinki institutional repository.

This is an electronic reprint of the original article.

This reprint may differ from the original in pagination and typographic detail.

Please cite the original version. 


\title{
SPONTANEOUSLY ARISING DISEASE
}

Short Title: Wooden Breast Myopathy in Broiler Chickens

Pectoral Vessel Density and Early Ultrastructural Changes in Broiler Chicken Wooden Breast Myopathy

\author{
H.-K. Sihvo* ${ }^{\dagger}$, N. Airas* , J. Lindén* and E. Puolanne ${ }^{\dagger}$ \\ ${ }^{*}$ Department of Veterinary Biosciences and ${ }^{\dagger}$ Department of Food and Environmental \\ Sciences, P.O. Box 66, FI-00014 University of Helsinki, Finland.
}

Correspondence to: H.-K. Sihvo (e-mail: hanna-kaisa.sihvo@helsinki.fi). 


\section{Summary}

In wooden breast myopathy (WBM) of broiler chickens, the pectoralis major muscles show abnormally hard consistency and microscopical myodegeneration of unknown aetiology. To date, previous studies have focused primarily on chronic WBM and ultrastructural descriptions of early WBM are lacking. The aim of this study was to elucidate the pathogenesis of WBM by light microscopical morphometry of vessel density and the ultrastructural description of early WBM changes with transmission electron microscopy. The pectoral vessel density was compared between unaffected chickens $(n=14)$ and two areas of focal WBM in affected chickens $(n=14)$. The transverse myofibre area per vessel was highest in the unaffected area of muscle from cases of focal WBM, significantly higher $(P=0.01)$ than in macroscopically unaffected tissue, indicating that relatively decreased blood supply may trigger the development of WBM. The ultrastructural study included unaffected chickens $(n=3)$, two areas of focal WBM from affected chickens $(n=3)$ and areas of diffuse WBM from affected chickens $(n=3)$. The morphologically least affected myofibres within the WBM lesion areas in light microscopy exhibited ultrastructural changes of increased sarcoplasmic reticulum diameter and mitochondrial hyperplasia. Such changes originate typically from osmotic imbalance, for which the most likely aetiologies in WBM include tissue hypoxia or myodegeneration of the surrounding myofibres. The findings suggest that a relative reduction of blood supply in the major pectoral muscle occurs in the early phase of WBM, which may be linked to the ultrastructural changes of osmotic imbalance.

Keywords: wooden breast myopathy; broiler chicken; vessel density; electron microscopy 


\section{Introduction}

Wooden breast myopathy (WBM) of broiler chickens manifests as a hardened consistency and pale colour of the major pectoral muscle and is characterized microscopically as polyphasic myodegeneration, accompanied by fibrosis in the chronic phase (Sihvo et al., 2014). WBM starts to develop as a focal lesion at approximately 2 weeks of age (Sihvo et al., 2017), but the aetiology and pathogenesis are currently unknown. In slaughter-age broilers of approximately 5-6 weeks of age, the WBM lesion has typically progressed into a chronic and diffuse phase, completely affecting the major pectoral muscle.

Recent hypotheses for WBM aetiopathogenesis include tissue hypoxia and oxidative stress; the genes associated with these conditions show increased expression in chronic cases of WBM (Mutryn et al., 2015; Abasht et al., 2016). Other studies of chronic WBM suggest differences in connective tissue composition as an aetiology or point to a multifactorial aetiology including altered glucose metabolism, unbalanced calcium ion homeostasis, disturbed muscle fibre development and others (Velleman and Clark, 2015; Zambonelli et al., 2016). However, chronic WBM involves prominent tissue repair processes and secondary changes, such as fibrosis (Sihvo et al., 2014). Accordingly, an analysis of the early changes in WBM in younger birds would be of greater value in elucidating the pathogenesis of WBM. Recently, the role of lymphocytic phlebitis in WBM has been speculated (Sihvo et al., 2017; Papah et al., 2017).

The major pectoral muscle of broiler chickens consists almost entirely of fast glycolytic type II b fibres (Remignon et al., 1995; Papinaho et al., 1996; MacRae et al., 2006). In rapidly growing high-yield broilers, the type II b fibres show increased diameter (hypertrophy) compared with slower-growing broilers or layer chickens (Remignon et al., 1995; Soike and Bergmann, 1998a; MacRae et al., 2006; Velleman and Clark, 2015; Clark and Velleman, 2016). Large muscle fibre diameter is speculated to link the high occurrence 
of muscle pathologies in high-yielding broilers to metabolic stress in the muscle. This is due to an increased diffusion distance for oxygen and other metabolites from the blood vessels to the centre of the myofibre (Soike and Bergmann, 1998a).

In addition to the higher initial myofibre diameter compared with layers, the myofibre diameter increases with age in high-yielding broilers, while the total vessel density decreases (Joiner et al., 2014; Radaelli et al., 2017). Capillaries, rather than other vessels, appear to increase in number in response to the myofibre hypertrophy in order to maintain the necessary blood supply to growing muscle (Joiner et al., 2014; Radaelli et al., 2017). Insufficient vessel number, either initially or due to the failure to increase the vessel number following myofibre hypertrophy, could further promote oxidative stress in the muscle. A definite relationship between microvessel number and WBM, however, remains to be established.

Currently, both the acute (Sihvo et al., 2017; Papah et al., 2017) and chronic lesions of WBM (Sihvo et al., 2014, 2017; de Brot et al., 2016; Soglia et al., 2016) have been described at the level of light microscopy, but ultrastructural descriptions of WBM and broiler skeletal muscle in general are sparse (Soike and Bergmann, 1998b; Papah et al., 2017). Ultrastructural changes associated with degeneration of skeletal muscle in general are well known. The first alterations include reduced myofibrillar width with normal sarcomere structure retained, while more progressed degeneration is characterized by Z-line alterations, destruction of sarcomeres and increased amounts of sarcoplasm that replace the sarcomeres (Ghadially, 1997a). These ultrastructural degenerative changes do not directly suggest any specific aetiopathogenesis, but electron microscopy is a valuable tool to exclude certain muscle diseases and to gather indirect evidence of metabolic or oxidative stress.

The aims of the present study were to evaluate microvessel density in the major pectoral muscle in unaffected chickens and chickens with focal WBM by light microscopy 
and to describe the ultrastructure of early WBM by transmission electron microscopy by comparing the unaffected chickens with birds having focal and diffuse WBM.

\section{Materials and Methods}

\section{Animals and Macroscopic Evaluation}

The birds of this study originated from two flocks (A and B) of high-yielding and fastgrowing, commercial hybrid broiler chickens. The birds, 240 male broiler chickens in flock A and 350 in flock B, were transferred from a commercial hatchery to an experimental rearing facility of the University of Helsinki within their first day of life. The unvaccinated 1-day-old birds were randomly divided in woodchip-bedded pens, 10 (flock A) or 14 birds (flock B) per pen, and provided with water and feed ad libitum. The base of the feed consisted of wheat and soya bean and $0.007 \%$ narasin (Monteban, Elanco, Greenfield, Indiana, USA), which was included as a coccidiostat. The lighting program included $6 \mathrm{~h}$ of darkness daily, except for the first 7 days when heating lamps were used. The broilers were weighed, killed by mechanical cervical dislocation and exsanguinated via cervical blood vessels before necropsy examination and macroscopical evaluation for WBM as previously described (Sihvo et al., 2017). Briefly, the major pectoral muscles were graded as unaffected (normal muscle consistency), focal WBM (muscle consistency hardened in a demarcated area, surrounded by areas of normal consistency) or diffuse WBM (the complete muscle area hardened). Since the birds were not subject to ante-mortem experimental procedures, legislation applicable to conventional breeding was followed in rearing and killing of the birds. The methods of rearing and killing were approved by the Laboratory Animal Centre of the University of Helsinki. 


\section{Microvessel Density}

The microvessel density study included 28 birds from flock A. The stratified random selection of the 28 broilers (14 unaffected and 14 with focal WBM) was at the age of 18 days $(n=3), 24$ days $(n=5), 35$ days $(n=4)$ and 38 days $(n=2)$. The right major pectoral muscle was sampled in the middle area (unaffected chickens) or in the lesion area and in the unaffected area (chickens with focal WBM), approximately $1 \mathrm{~cm}$ deep from the skin-facing surface (Fig. 1). Chickens with diffuse WBM were not included due to the advanced microscopical tissue destruction and secondary changes. Tissue samples of $1 \times 1 \times 2 \mathrm{~cm}$ were fixed in $10 \%$ neutral buffered formalin without pinning, dehydrated through an ascending series of alcohols and embedded in paraffin wax. Sections $(4 \mu \mathrm{m})$ were stained with haematoxylin and eosin (HE) for histological confirmation of WBM or by the diastase periodic acid-Schiff reaction (PASD) to visualise the basement membrane of blood vessels and endomysium. All tissue sections were graded for myodegeneration on a scale of absent to minimal, mild, moderate or severe (Sihvo et al., 2017). The sections were coded with a random number to avoid sampling area bias.

For each sample, eight microscopical fields $\left(0.15 \mathrm{~mm}^{2}\right.$ each $)$ of PASD-stained sections were photographed (AxioLabA1 microscope and Axiovision software, Carl Zeiss, Jena, Germany) in areas representative of the typical morphology of the tissue section, but devoid of large vessels. The number of microvessels (up to approximately 50-70 $\mu \mathrm{m}$ in diameter) and myofibres were counted manually from each photograph; entirely degenerate myofibres with a disrupted endomysium were excluded. Each myofibre that was incompletely present in the field of view (i.e. at the edge of the photograph) counted as half a fibre. The coefficient of variation for vessel and myofibre number was determined for each set of eight photographs. 
From each set of eight photographs, two were selected for determination of myofibre area. The selection was random after exclusion of the photographs that featured the smallest and the highest myofibre number in the manual myofibre count in each set of eight photographs. Random numbers were assigned for the remaining six photographs and two photographs with the smallest random numbers were selected. For each complete myofibre (i.e. incomplete fibres at the edges of the photograph were excluded), the cross-sectional area was calculated in a semi-automated fashion with the spline contour function of ZEN Pro software (Carl Zeiss).

For each set of two photographs, the microvessel number was combined (total vessel number) and the area of myofibres was combined (total myofibre area). The ratio of these summed parameters was calculated (total myofibre area per total vessel number). Only the sets of two microscopical fields were included in further statistical analyses. One-way ANOVA with Bonferroni post-hoc analysis was performed to test the differences in vessel number or myofibre area between the unaffected, focal WBM unaffected and focal WBM lesion areas. Based on the ANOVA results, the birds were pooled into two age groups and the total myofibre area, total vessel number and the ratio of those two were analysed with t-tests. Statistical analyses were performed with IBM SPSS Statistics (v.24, IBM Corp., Armonk, New York, USA) and $P<0.05$ was considered significant.

\section{Ultrastructure}

The ultrastructural study included nine broilers that were retrieved with a stratified random procedure (three unaffected, three with focal WBM and three with diffuse WBM) from the 47 broilers that were killed at 22 days of age out of the 350 birds in flock B. In order to obtain samples from early WBM lesions, the ultrastructural study was targeted at 22-day-old birds that presented with both focal and diffuse forms of WBM, but had not yet developed fibrosis 
and other prominent secondary changes (Sihvo et al., 2017). After death and WBM evaluation and within 5 min post-mortem, tissue samples for light and electron microscopy were collected from the right major pectoral muscle of the nine broilers according to the macroscopical appearance (Fig. 1). A $1 \times 1 \times 2 \mathrm{~cm}$ sample was obtained from each area for histological confirmation of the WBM diagnosis by light microscopy. The samples were formalin-fixed and processed routinely. Sections $(4 \mu \mathrm{m})$ were stained with HE and evaluated with observers being blinded to the macroscopical results. All tissue sections were graded for myodegeneration on a scale of absent to minimal, mild, moderate or severe (Sihvo et al., 2017).

Several $2 \times 2 \times 2 \mathrm{~mm}$ tissue samples from each area (Fig. 1) were obtained for transmission electron microscopical analysis. The samples were immersed in $2.5 \%$ glutaraldehyde (Sigma-Aldrich, St. Louis, Missouri, USA) at pH 7.4 and room temperature for $2 \mathrm{~h}$ then immersed in $2 \%$ paraformaldehyde (Sigma-Aldrich) at $4^{\circ} \mathrm{C}$. Two of the tissue samples were further post-fixed with 1\% osmium tetroxide (Electron Microscopy Sciences, Hatfield, England) for $1 \mathrm{~h}$ at room temperature, dehydrated using a graded series of ethanols (70\%, 96\% and 100\%), incubated with transitional solvent acetone and then embedded gradually in Epon (TAAB Laboratories Equipment Ltd., Aldermaston, England). Toluidine blue-stained semithin $(500 \mathrm{~nm})$ sections of longitudinally oriented samples were examined under a light microscope to select areas for preparation of $70 \mathrm{~nm}$ ultrathin sections. For ultrathin sections, areas of severe myodegeneration were avoided and areas with intact myofibres without marked degeneration were selected to assess early ultrastructural changes. Electron micrographs were acquired using a Jeol JEM-1400 electron microscope (Jeol Ltd., Tokyo, Japan) and Gatan Orius SC 1000B bottom-mounted CCD camera (Gatan Inc., Pleasanton, California, USA). Sarcoplasmic reticulum size variation between areas was assessed using diameter measurements performed from the micrographs (approximately 28 
micrographs per each sample area). The transversal diameter of all visible sarcoplasmic reticulum pouches were measured, results were expressed rounded to the nearest $100 \mathrm{~nm}$ and the rounded values were ranked without statistical analysis.

\section{Results}

\section{Animals and Evaluation of Wooden Breast Myopathy}

The birds included in this study showed no clinical signs or significant post-mortem changes other than WBM. Average daily weight gain was $65 \mathrm{~g}$ (flock $\mathrm{A}, 42$ days) and $71 \mathrm{~g}$ (flock B, 43 days). However, due to different number of animals killed at various ages, the average daily weight gains between flocks A and B are not directly comparable. Mortality rates of $5.8 \%$ for flock A and 5.7\% for flock B occurred over the entire rearing period. The most common post-mortem changes were typical for ascites syndrome and sudden death syndrome (Klasing et al., 2008).

The macroscopical appearance of the major pectoral muscle was similar between the cases within each group (unaffected, focal WBM and diffuse WBM). The unaffected chickens did not exhibit changes, the chickens with focal WBM had a variablysized, hardened and pale area that covered the cranial half of the major pectoral muscle at maximum, while in the chickens with diffuse WBM, these changes covered the complete muscle. Histologically, the tissue morphology of the HE-stained sections corresponded with the macroscopical diagnosis of WBM. The unaffected tissue and the unaffected area of tissue with focal WBM featured absent to minimal or mild myodegeneration, while the lesional areas of focal WBM and diffuse WBM exhibited moderate or severe myodegeneration. Other morphological changes typical for WBM, such as myofibre separation and shape aberration, loose connective tissue accumulation and perivascular lymphoid aggregates were consistent 
with the degree of myodegeneration; the higher the degree of myodegeneration, the more pronounced the changes were in general. However, all young birds (i.e. 18, 22 or 24 days old) lacked prominent fibrosis, even those affected severely.

\section{Microvessel Density}

The microvessel density was evaluated from the PASD-stained sections. The representative nature of two microscopical fields out of eight was verified by calculating the coefficient of variation for vessel and myofibre number for each set of eight fields. The median coefficient of variation was $9.2 \%$ for vessel number and $11.2 \%$ for myofibre number.

Further tests were performed on the summed vessel number and myofibre areas of the two selected microscopical fields only. One-way ANOVA showed a significant difference in vessel number $(P<0.001)$ and myofibre area $(P=0.002)$ when the sampling areas (unaffected, focal WBM unaffected and focal WBM lesion area) for each age group were combined. However, Bonferroni post-hoc analysis indicated no significant difference between the 18- and 24-day-old birds and between the 35- and 38-day-old birds, but the former were significantly different from the latter two ages (Total vessel number: 18 versus 35 days, $P<0.001 ; 18$ versus 38 days, $P=0.001 ; 24$ versus 35 days, $P=0.001 ; 24$ versus 38 days, $P=0.007$. Total myofibre area: 18 versus 35 days, $P=0.017 ; 18$ versus 38 days, $P=$ $0.036 ; 24$ versus 35 days, $P=0.018 ; 24$ versus 38 days, $P=0.049$ ).

Based on the ANOVA results, the birds were pooled into younger (18 and 24 days old) and older (35 and 38 days old) age groups and further statistical tests were conducted with these groups. The total myofibre area, total vessel number and their ratio were compared between the areas (unaffected, focal WBM unaffected, focal WBM lesional tissue); independent t-test with Bonferroni multiple comparison was applied to compare unaffected with focal WBM unaffected and with focal WBM lesional tissue, while a paired t- 
test was performed to compare the focal WBM unaffected and lesional areas. Capillaries constituted the majority of microvessels, while other types of microvessels (i.e. arterioles and venules) were in the minority, but were observed in all microscopical fields. The detailed ttest results for the pooled age groups are presented in Fig. 2. Briefly, the main significant difference in the vessel number was observed in the group of younger birds (18 and 24 days of age), where the unaffected tissue exhibited significantly $(P<0.001)$ higher mean vessel number than tissue from the two sites of chickens with focal WBM. The myofibre area per microscopical field was reduced with the progression of WBM; the total myofibre area was significantly higher in the unaffected young birds than in the unaffected area of chickens with focal WBM $(P=0.02)$ and the lesional area of chickens with focal WBM $(P=0.006)$. In the older age group, the myofibre area was significantly reduced in the WBM lesional area, compared with the unaffected tissue $(P=0.002)$ or unaffected tissue of chickens with focal WBM $(P=0.005)$. The ratio of myofibre area per vessel number was significantly lower in the unaffected tissue compared with tissue from the unaffected area of chickens with focal WBM in both young and older age groups $(P=0.01$ and $P=0.04$, respectively).

\section{Ultrastructure}

The toluidine blue-stained semithin sections, evaluated under a light microscope, displayed degenerative myofibres and other typical changes of WBM, corresponding to the macroscopical and histological diagnosis. The unaffected tissues and unaffected areas of tissue from chickens with focal WBM exhibited absent or minimal changes. In contrast, in the tissue from chickens with diffuse WBM and in the focal WBM lesional areas, variable numbers of myofibres exhibited irregular width, fragmentation, separation from each other, loss of cross-striation (i.e. myofibre degeneration) and increased amounts of loose connective tissue between the myofibres. However, our aim was to observe the early morphological 
alterations in WBM, therefore we selected the least-changed areas for the preparation of ultrathin sections.

Transmission electron microscopy of the ultrathin sections revealed that the most remarkable changes between the unaffected tissue and focal and diffuse WBM existed in the sarcoplasmic reticulum and mitochondria. The most common diameter of the sarcoplasmic reticulum was approximately $100 \mathrm{~nm}$ or less (Fig. 3a) in unaffected tissue and unaffected areas of tissue from chickens with focal WBM, while a typical diameter for the focal WBM lesional areas and the diffuse WBM areas was in the 200-300 nm range. The maximum observed diameter was $300 \mathrm{~nm}$ in unaffected tissue, $400 \mathrm{~nm}$ in tissue from chickens with focal WBM (Fig. 3b) and $600 \mathrm{~nm}$ in tissue from chickens with diffuse WBM. The enlargement affected both forms of sarcoplasmic reticulum: free sarcoplasmic reticulum and terminal cisternae that surround the T-tubules. The lumen of the terminal cisternae often contained granular material of high electron density, while the free sarcoplasmic reticulum generally displayed a completely electron-lucent lumen. However, the terminal cisternae also occasionally featured electron-lucent empty lumina, especially when their diameters were enlarged. The triads, composed of one T-tubule flanked by terminal cisternae on both sides (Fig. 3a), were typically located at the Z-line, but some triads were closer to the junction of the A- and I-bands (Fig. 3c), irrespective of the WBM status.

The intermyofibrillar mitochondria of the unaffected tissue and unaffected areas of tissue from chickens with WBM typically appeared singly or in rows of two to three aligned along the myofibrils. The tissue from chickens with diffuse WBM and the lesional area of focal WBM additionally exhibited aggregations of 10 to 25 mitochondria between the myofibrils (i.e. mitochondrial hyperplasia, Fig. 3d), with occasional aggregations of $>40$ mitochondria in the tissue from chickens with diffuse WBM. Vacuolation, swollen matrix chamber or loss of cristae (Fig. 3d) were apparent in the mitochondria of all cases, both in 
mitochondria occurring singly or in aggregations. These changes to the mitochondria were the most frequent and prominent in diffuse WBM and in the lesional area of focal WBM. The degree of mitochondrial changes varied from minimal to severe even within one myofibre and both unaltered and altered mitochondria were present in all cases. Swollen sarcoplasmic reticulum and morphologically altered mitochondria appeared typically in conjunction with each other, but unaltered mitochondria were also observed among areas with swollen sarcoplasmic reticulum (Fig. 3c), and vice versa. The subsarcolemmal mitochondria exhibited vacuolation to variable degrees, without marked differences in their numbers or in morphological changes between the three groups as indicated by subjective assessment.

Although we selected the least-degenerate areas of the semithin sections for the ultrastructural evaluation, a few myofibres of the samples from chickens with diffuse WBM and of the lesional area of tissue from chickens with focal WBM exhibited reduced myofibrillar width with normal sarcomere structure, indicative of early myofibrillary degeneration (Fig. 3e). Otherwise, the sarcomere structure was well preserved in the selected myofibres and showed no characteristic features indicative of pronounced myofibre degeneration or sarcomere-origin diseases, such as sarcomere disorientation, Z-line aberrations, ring fibres, central cores or lipid accumulation. The examined myofibres contained no nemalin rods or inclusion bodies characteristic of some hereditary myopathies. The myocyte nuclei activity typically varied and no perinuclear lipofuscin accumulation was observed. Glycogen granules were present in the typical locations in all nine broilers. Approximately $60 \mathrm{~nm}$ vesicular invaginations of the plasma membrane (Fig. 3f), morphologically resembling caveolae, were observed in the sarcolemma of all three groups (i.e. unaffected, focal and diffuse WBM).

\section{Discussion}


This study explored the pathogenesis of WBM by light microscopical evaluation of pectoral microvessel density in unaffected tissue and tissue from young and slaughter-age broiler chickens with focal WBM and by transmission electron microscopy of unaffected tissue and tissue from 22-day-old broiler chickens with focal and diffuse WBM.

The total vessel number decreased with age in unaffected broiler chickens (Fig. 2), which is consistent with previous findings (Joiner et al., 2014; Radaelli et al., 2017). Between the two areas of tissue from birds with focal WBM (i.e. unaffected and lesional area), the vessel number was comparable in both younger and slaughter-age broilers, but significantly different from the unaffected young group. This finding suggests that the highest reduction in vessel number takes place during the early initiation phase of WBM, before the macroscopical lesion exists, or that the vessel density is initially reduced in birds that will succumb to WBM. The total vessel number of the focal WBM lesional area likely includes newly formed blood vessels (i.e. neovascularization) in addition to pre-existing vessels, especially in the slaughter-age broilers. The total myofibre area decreased from unaffected tissue to lesional tissue from birds with focal WBM, associated with the corresponding myodegeneration seen histologically.

The myofibre area to vessel number ratio followed a similar pattern both in the young and slaughter-age broilers; the ratio was significantly lower in the unaffected tissue compared with the unaffected area of tissue from birds with focal WBM, which exhibited the highest myofibre area per vessel number. These findings indicate that during the early phase of WBM lesion development, the blood supply per myofibre may be compromised and may provoke further progression of the WBM lesion. The macroscopically unaffected area of tissue from birds with focal WBM likely represented a transition phase in WBM lesion development, since reduced vessel density and mild histological myodegeneration was present, but no macroscopical lesion existed. Contrary to the usual evaluation of the capillary 
supply of muscle tissue only as capillary density (i.e. number of capillaries per $\mathrm{mm}^{2}$ area) or capillary-to-myofibre ratio (i.e. number of capillaries per number of muscle fibres) (Hudlická, 1985; Hoving-Bolink et al., 2000), we used the myofibre area to vessel number ratio in vascular assessment. This was to correct the bias in myofibre number variation between unaffected and WBM tissue; the muscle architecture in WBM lesional areas is disrupted and myofibre size varies due to degeneration and regeneration (Soike and Bergmann, 1998b; Sihvo et al., 2014, 2017; de Brot et al., 2016; Soglia et al., 2016).

The 22-day-old broilers were optimal for the ultrastructural study, as they expressed both focal and diffuse WBM phases before the establishment of noticeable fibrosis and other features of advanced chronicity. We observed typical macroscopical and histological changes of WBM in the affected major pectoral muscles. However, we selected the non-degenerate myofibres within the lesion for electron microscopical evaluation to detect and characterize the early-phase morphological changes that precede myodegeneration. In both types of WBM lesions (i.e. focal and diffuse), the non-degenerate myofibres featured increased diameter of the sarcoplasmic reticulum. Dilation of the sarcoplasmic reticulum results from the entrance of excess water into the cell due to osmotic imbalances, which may occur as a primary sarcoplasmic defect or secondary to other processes, such as myodegeneration, fatigue or hypoxia (Fujisawa, 1975; Ghadially, 1997b; Duhamel et al., 2004; Frías et al., 2005). Hypoxia reduces calcium ion transfer from the cytoplasm into the sarcoplasmic reticulum due to downregulation of sarco-endoplasmic reticulum $\mathrm{Ca}^{2+}$-ATPase, but muscle fatigue also seems to stem from impaired calcium ion regulation (Duhamel et al., 2004; Frías et al., 2005; Revuelta-López et al., 2015). Muscle fatigue plausibly enhances the progression of a well-pronounced WBM lesion, since the remaining functional myofibres probably endure an increased workload due to degeneration and detachment of the surrounding fibres. However, the role of muscle fatigue in the initiation and 
aetiopathogenesis of the WBM process is currently unknown. Defective calcium ion regulation in WBM is further suggested by the reduced luminal electron density and granularity of some enlarged terminal cisternae. The granular material within the lumen of terminal cisternae is likely calsequestrin, a protein that enhances the calcium-binding capacity of the sarcoplasmic reticulum (Cheville, 2009; Franzini-Armstrong and Engel, 2012).

The morphology of the intermyofibrillar mitochondria of all three of our broiler groups (i.e. unaffected, focal and diffuse WBM) varied from minimal to pronounced changes including vacuolation, loss of cristae and swelling. However, the most severe changes were more abundant in the tissue from birds with diffuse WBM and in the lesional areas of tissue from birds with focal WBM. Mitochondrial swelling, vacuolation and cristae loss are typical consequences of osmotic imbalance and hypoxia, especially in cells with high metabolic needs (Sewry, 2002; Cheville, 2009). This indicates that the WBM lesional areas either experienced distress (such as hypoxia during life) or are more prone to post-mortem alterations.

In addition to the structural alterations, mitochondrial aggregates indicative of hyperplasia appeared in areas affected by WBM. Mitochondrial hyperplasia or hypertrophy, or both, occur in various pathologies and during increased functional burden to the skeletal muscle myofibres (Ghadially, 1997c). Hypoxic conditions increase especially in intermyofibrillar mitochondrial density and volume in human skeletal muscle, while the aggregation of mitochondria is also associated with myofibrillar loss (Sewry, 2002; Jacobs et al., 2016). In WBM, myodegeneration surrounded the myofibres that we studied and this potentially impacts on mitochondrial hyperplasia, but as hypoxia may induce similar changes, the effect of those two factors cannot be differentiated ultrastructurally. The presence of caveolae-like sarcolemmal invaginations, both in the unaffected and WBM tissues in our study, indicated that caveolin deficiency can be ruled out as an aetiology of WBM. 
Electron microscopical analyses are prone to artefacts. The mitochondria and sarcoplasmic reticulum are especially sensitive to artefactual enlargement, but pathological swelling may also exacerbate the artefactual changes when the two processes superimpose (Sewry, 2002; Cheville, 2009). Although swollen sarcoplasmic reticulum and mitochondria were observed in all three groups of our ultrastructural study, well-conserved mitochondria were also present within the morphologically altered areas of tissues with diffuse and focal WBM, which indicates the pathological origin of the changes. A pathological process is further supported by the increased enlargement of the sarcoplasmic reticulum and greater severity of mitochondrial morphological alterations found in the areas of focal and diffuse WBM compared with the unaffected tissue and unaffected tissue from affected chickens, which underwent identical sampling and processing procedures. Surrounding myodegeneration may provoke ultrastructural changes in the non-degenerate myofibres in the focal lesional areas and in diffusely affected tissue. However, as myodegeneration is an essential component of WBM diagnosis, it is practically impossible to avoid it in studies of WBM. In addition, the extensive cell length of the myocytes makes the evaluation of the complete myocyte by electron microscopy impossible; the non-degenerate myocytes may have endured distress outside the field of view.

In conclusion, the current study provides insight into the possible linkage of reduced vessel density and ultrastructural alterations in early WBM. Our results show that the myofibre area per blood vessel is the highest in the unaffected area of tissue from chickens with focal WBM, suggesting that the major pectoral muscle may suffer from relatively reduced microvessel supply during the initiation of WBM lesion development. Electron microscopical evaluation revealed that non-degenerate myofibres among the WBM lesions have an increasingly swollen sarcoplasmic reticulum and hyperplasia and morphological mitochondrial alterations when compared with myofibres of macroscopically unaffected 
areas. These changes are known artefacts in ultrastructural studies, but as tissue from all nine broilers was processed similarly, they appear to reflect true ante-mortem changes in broiler chickens with WBM, further suggesting hypoxia as one possible mechanism. However, a quantitative study with a higher number of chickens is necessary to further confirm our findings.

\section{Acknowledgments}

The authors thank Professor E. Lehtonen for valuable comments on electron microscopy. The current address of H.-K. Sihvo is Finnish Food Safety Authority Evira, Veterinary Bacteriology and Pathology Research Unit, Mustialankatu 3, 00790 Helsinki, Finland. This work was supported by the Finnish Foundation of Veterinary Research (grant number 10-868-24) and the Niemi Foundation (grant number 161015).

\section{References}

Abasht B, Mutryn MF, Michalek RD, Lee WR (2016) Oxidative stress and metabolic perturbations in wooden breast disorder in chickens. PLoS ONE, 11, e0153750.

Cheville NF (2009) Ultrastructural Pathology: The Comparative Cellular Basis of Disease, $2^{\text {nd }}$ Edit., Wiley-Blackwell, Ames, pp. 75-198.

Clark DL, Velleman SG (2016) Spatial influence on breast muscle morphological structure, myofiber size, and gene expression associated with the wooden breast myopathy in broilers. Poultry Science, 95, 2930-2945.

de Brot S, Perez S, Shivaprasad HL, Baiker K, Polledo L et al. (2016) Wooden breast lesions in broiler chickens in the UK. Veterinary Record, 178, 141. 
Duhamel TA, Green HJ, Sandiford SD, Perco JG, Ouyang J (2004) Effects of progressive exercise and hypoxia on human muscle sarcoplasmic reticulum function. Journal of Applied Physiology, 97, 188-196.

Franzini-Armstrong C, Engel A (2012) Skeletal muscle: architecture of membrane systems. In: Muscle, EN Olxon, J Hill, Eds., Academic Press, London, pp. 763-772.

Frías JA, Cadefau JA, Prats C, Morán M, Megías A et al. (2005) Disturbances of the sarcoplasmic reticulum and transverse tubular system in 24-h electrostimulated fast-twitch skeletal muscle. Biochimica et Biophysica Acta, 1668, 64-74.

Fujisawa K (1975) Some observations on the skeletal musculature of aged rats. Part 2. Fine morphology of diseased muscle fibres. Journal of Neurological Sciences, 24, 447-469.

Ghadially FN (1997a) Intracytoplasmic filaments. In: Ultrastructural Pathology of the Cell and Matrix, $4^{\text {th }}$ Edit., Butterworth-Heinemann, Newton, pp. 887-982.

Ghadially FN (1997b) Endoplasmic reticulum. In: Ultrastructural Pathology of the Cell and Matrix, $4^{\text {th }}$ Edit., Butterworth-Heinemann, Newton, pp. 433-582.

Ghadially FN (1997c) Mitochondria. In: Ultrastructural Pathology of the Cell and Matrix, $4^{\text {th }}$ Edit., Butterworth-Heinemann, Newton, pp. 195-328.

Hoving-Bolink AH, Kranen RW, Klont RE, Gerritsen CL, de Greef KH (2000) Fibre area and capillary supply in broiler breast muscle in relation to productivity and ascites. Meat Science, 56, 397-402. 
Hudlická O (1985) Development and adaptability of microvasculature in skeletal muscle.

Journal of Experimental Biology, 115, 215-228.

Jacobs RA, Lundby AM, Fenk S, Gehrig S, Siebenmann C et al. (2016) Twenty-eight days of exposure to $3454 \mathrm{~m}$ increases mitochondrial volume density in human skeletal muscle. Journal of Physiology, 594, 1151-1166.

Joiner KS, Hamlin GA, Lien AR, Bilgili SF (2014) Evaluation of capillary and myofiber density in the pectoralis major muscles of rapidly growing, high-yield broiler chickens during increased heat stress. Avian Diseases, 58, 377-382.

Klasing K, Crespo R, Shivaprasad H, Hoerr F, Fulton R (2008) Non-infectious diseases. In: Diseases of Poultry, 12 ${ }^{\text {th }}$ Edit., YM Saif, AM Fadly, JR Glisson, LR McDougald, LK Nolan et al., Eds., Wiley-Blackwell, Ames, pp. 1121-1258.

MacRae VE, Mahon M, Gilpin S, Sandercock DA, Mitchell MA (2006) Skeletal muscle fibre growth and growth associated myopathy in the domestic chicken (Gallus domesticus). British Poultry Science, 47, 264-272.

Mutryn MF, Brannick EM, Fu W, Lee WR, Abasht B (2015) Characterization of a novel chicken muscle disorder through differential gene expression and pathway analysis using RNA-sequencing. BMC Genomics, 16, 399.

Papah MB, Brannick EM, Schmidt CJ, Abasht B (2017) Evidence and role of phlebitis and lipid infiltration in the onset and pathogenesis of wooden breast disease in modern broiler chickens. Avian Pathology, 46, 623-643. 
Papinaho PA, Ruusunen MH, Suuronen T, Fletcher DL (1996) Relationship between muscle biochemical and meat quality properties of early deboned broiler breasts. Journal of Applied Poultry Research, 5, 126-133.

Radaelli G, Piccirillo A, Birolo M, Bertotto D, Gratta F et al. (2017) Effect of age on the occurrence of muscle fiber degeneration associated with myopathies in broiler chickens submitted to feed restriction. Poultry Science, 96, 309-319.

Remignon H, Gardahaut MF, Marche G, Ricard FH (1995) Selection for rapid growth increases the number and the size of muscle fibres without changing their typing in chickens. Journal of Muscle Research and Cell Motility, 16, 95-102.

Revuelta-López E, Cal R, Herraiz-Martínez A, de Gonzalo-Calvo D, Nasarre L et al. (2015) Hypoxia-driven sarcoplasmic/endoplasmic reticulum calcium ATPase 2 (SERCA2) downregulation depends on low-density lipoprotein receptor-related protein 1 (LRP1)-signalling in cardiomyocytes. Journal of Molecular and Cellular Cardiology, 85, 25-36.

Sewry CA (2002) Electron microscopy of human skeletal muscle: role in diagnosis. Current Diagnostic Pathology, 8, 225-231.

Sihvo HK, Lindén J, Airas N, Immonen K, Valaja J et al. (2017) Wooden breast myodegeneration of pectoralis major muscle over the growth period in broilers. Veterinary Pathology, 54, 119-128.

Sihvo HK, Immonen K, Puolanne E (2014) Myodegeneration with fibrosis and regeneration in the pectoralis major muscle of broilers. Veterinary Pathology, 51, 619-623. 
Soglia F, Mudalal S, Babini E, Di Nunzio M, Mazzoni M et al. (2016) Histology, composition, and quality traits of chicken pectoralis major muscle affected by wooden breast abnormality. Poultry Science, 95, 651-659.

Soike D, Bergmann V (1998a) Comparison of skeletal muscle characteristics in chicken bred for meat or egg production. II. Histochemical and morphometric examination. Journal of Veterinary Medicine Series A, 45, 169-174.

Soike D, Bergmann V (1998b) Comparison of skeletal muscle characteristics in chicken bred for meat or egg production. I. Histopathological and electron microscopic examination. Journal of Veterinary Medicine Series A, 45, 161-167.

Velleman SG, Clark DL (2015) Histopathologic and myogenic gene expression changes associated with wooden breast in broiler breast muscles. Avian Diseases, 59, 410-418.

Zambonelli P, Zappaterra M, Soglia F, Petracci M, Sirri F et al. (2016) Detection of differentially expressed genes in broiler pectoralis major muscle affected by white striping - wooden breast myopathies. Poultry Science, 95, 2771-2785.

Received, June $12^{\text {th }}, 2017$

Accepted,

Figure Legends 
Fig. 1. Major pectoral muscles of unaffected broiler chickens and chickens with focal and diffuse WBM. The solid black dot depicts a sampling area for the light and electron microscopical evaluations of tissue morphology. The grey colour represents the maximum macroscopical WBM lesional area. WBM, wooden breast myopathy; PASD, diastase periodic acid-Schiff reaction.

Fig. 2. Total microvessel number, total myofibre area and their ratio in two microscopical fields of major pectoral muscle of young (18 and 24 days old; $n=16$; eight unaffected chickens and eight chickens with focal WBM) and slaughter-age (35 and 38 days old; $n=12$; six unaffected chickens and six chickens with focal WBM) broiler chickens. Bars represent mean and $95 \%$ confidence interval. $P$ values refer to t-test results subjected to Bonferroni correction $(\alpha / 2)$.

Fig. 3. Transmission electron micrographs of major pectoral muscle of broiler chickens. (A) Terminal cisternae (arrows) of the triad complex exhibit an approximately $100 \mathrm{~nm}$ diameter at the Z-line of the myofibril below, while the free sarcoplasmic reticulum remains inconspicuous between the glycogen granules (arrowhead). Unaffected chicken. Bar, $200 \mathrm{~nm}$. (B) Sarcoplasmic reticulum with an electron-lucent lumen displays a diameter up to $400 \mathrm{~nm}$ (arrow). Chicken with diffuse WBM. Bar, $200 \mathrm{~nm}$. (C) Singly-appearing intermyofibrillar mitochondrion displays minimal structural defects and the sarcoplasmic reticulum (arrow) is slightly swollen. A triad (arrowhead) is located between the Z-line and the junction of the Aand I-bands. The lesional area of a chicken with focal WBM. Bar, 200 nm. (D) Aggregation of intermyofibrillar mitochondria that express vacuolation (arrows), swelling and loss of cristae, while one mitochondrion remains minimally altered (double arrow). Sarcoplasmic reticulum (asterisks) varies in diameter. Chicken with diffuse WBM. Bar, $1 \mu \mathrm{m}$. (E) 
Myofibrils of a variable width, slightly separated from each other and exhibiting slight Z-line aberrations, were occasionally observed in the WBM lesions. An intermyofibrillar mitochondrion exhibits partial vacuolation and disruption of the cristae. The lesional area of a chicken with focal WBM. Bar, $1 \mu \mathrm{m}$. (F) Caveolae-like invaginations (arrows) on the sarcolemma were present both in unaffected muscle and muscle from chickens with WBM. Lesional area of a chicken with focal WBM. Bar, $200 \mathrm{~nm}$. 